\title{
Analysis of The Smart Device Invention for The Detection and Repair of Asphalt Road Surface Holes
}

\author{
Mohammad Hersh Baghadam \\ Technical and Vocational University, Iran
}

\begin{abstract}
Detecting, finding and repairing road surface holes is an important issue in road maintenance. This device recognizes the in-depth intelligence of the holes on the surface of the road, and the electronic control unit controls the ready and leveling operators. The main chassis is a platform with components on top of it, such as the rear-trailer, with a diesel engine. The surface height sensors located at the beginning of the chassis scan the hole information. The data processing unit calculates the depth, and volume of the hole, and adjusts the location of all cutting, blasting, special slurry, Bitumen asphalt, and flattener and roller operators to suit the location of the holes. Then ECU sends the data information to the operators in sequence. To adjust the time of injection and the amount of motion of each operator, a certain algorithm is tailored to the dimensions of the hole by the processing unit. Once the track of the asphalt road is determining by this intelligent system, the paths are detected and recorded, and all of the holes will be restored with asphalt. Using this device reduces the time, costs, number and error of operator in roads maintenance. This innovative device was recorded in Iranian patent center.
\end{abstract}

Keywords: Actuator, Chassis, Dispenser, ECU, Sensor 


\section{Introduction}

Road transportation is one of the most important indicators of the development of human societies. Therefore, maintenance and maintenance of communication paths from public service areas is very important. Depreciation, overburdening, inappropriate substructure, chemical corrosively and other parameters are among the causes of the formation of the holes and pits in communication paths. It is important to maintain communication ways to provide safe and efficient transportation in a country. If the road network infrastructure is in violation, the transportation system will be disturbed and will cause a lot of consequences. Today's funding and budget constraints have caused road maintenance organizations to do more at a lower cost. The developmental and decision-making management centers in the field of the past had more emphasis on the strategy of building new ways, but now it is important to maintain and maintain the existing road pavement and choose the optimal maintenance method. This idea has resulted in three types of maintenance, preventive, corrective and emergency maintenance.

Detecting and repairing pits is a must-have for road maintenance. The procedure for detecting the cavity and repairing it has so far been that the hole was detected and identified by the human vision, then the preparation operations for asphalt and asphalt are also performed by the manpower with the help of the machines that are required by the operator. It should be noted that this process is timely and, most importantly, requires one-time track monitoring, one-time marking and, finally, one-time repair operations. The probability of visual error and performance error in this common methodology is a worrying technical problem in the field of road maintenance. The purpose of this plan is to mechanize all the steps and implement them in an integrated process.

The purpose of the invention is to use a device that intelligently detects the pits and cavities of the asphalt road and processes information and send orders to operators to prepare and fix the asphalt. One of the main goals of the invention is tracking one-off routes, identifying, detecting and recording the entire cavity and path pits, integrated path repair, reducing the number and error of manpower.

\section{Technical Problem, Cracks and Cavities in Asphalt}

Different types of road failures can be divided into two groups of fundamental failure and surface failure. The fundamental failure occurs when the pavement system is damaged due to inadequate load bearing capacity and can no longer withstand further loading without further increase in failure. Surface malfunction occurs when the pavement system is not necessarily lost in terms of its bearing strength structure, due to the overlapping surface of the pavement, it can 


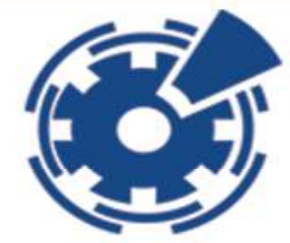

be exploited in some way. Identifying the type of fundamental and superficial failure is very important in terms of choosing the type and type of repair and repair of the pavement. To repair fundamental damage, the pavement system should be strengthened from a structural point of view. While surface defects must be repaired, they must be smooth and smooth with the necessary measures. Of course, in the division of another, the road's failure is divided into four major categories of cracks, the change in the surface of the pavement, the fragmentation of the asphalt and the sliding surface of the pavement.

The smearing operation is performed to repair the failure. Spotting is a process in which a range of pavements that are damaged greatly is removed and replaced with other materials to cover the damaged area. Sealing is also an important part of the maintenance of asphalt pavement. Leakage sealing reduces the penetration of water and incompressible material into the underlying layers, which ultimately leads to increased durability and survival of the pavement. At present, many methods are used to stave off cracking and cracking, and it is necessary to know what is in any situation what method and composition it is.

In general, the types of transverse, longitudinal, block, lumbar, edged and slippery cracks cause asphalt failure. Sometimes, in the process of smelting or weathering, the pavement seeds also become detached and cause cracks and cavities. But most of the time cracking is caused by changes in temperature, expansion and contraction of materials, and then expands due to the flexural stress resulting from loading and passing of high traffic loads on the layer. Occasionally crack is created by the stress of shear forces and horizontal surface tensions between the wheel and the surface of the pavement resulting from the loading and traffic flow, and then expands in the layer. A wave in asphalt is also a kind of failure that is caused by a wave in the direction of moving vehicles at the surface of the asphalt pavement. Another kind of breakdown is the pits and gaps that are caused by the fragmentation and fragmentation of a part of the material of the foundation of the pavement. All of these cracks and cracks lead to the creation of a hole in the road surface, which more needs to be repaired, restored and maintaine.

\section{Previous Knowledge and Current State of the Road Pit Repair}

In racking phenomenon is inevitable, without notice, that it will accelerate the breakdown in the surface of the pavement. Maintenance will help increase the durability of the pavement and create a smoother surface. Traditional sealing methods are different both in terms of equipment and in terms of the type of materials used with new sealing materials and equipment. In traditional methods, emulsion bitumen is used, bitumen or pure bitumen, and the equipment for the transfer of bitumen from a machine or hand. However, in modern methods, modified bitumen and using new equipment provides better conditions for performance. The modern method of sealing involves the restoration of cracking with local tapping to prevent the penetration of water and debris into the cracks, which may, in order to completely clear the 


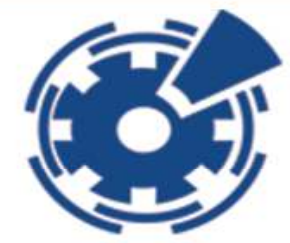

cracks and create a reservoir for the maintenance of the water-soluble material, including crack cutting operations Also be. In the past, the operation of the diagnosis and repair of the cavity of the pavement was completely traditional and by manpower. Over time, with the advancement of technology, the preparation of caverns and critical road ranges is carried out using compressor and compressor electric motors. Devices for the smelting of bitumen have also been developed for the operation of the smearing, which requires the operator.

For each of the processes of pouring, flattening, reinforcing and rolling of asphalt, there are also separate, sometimes coherent, devices. But in the operation to diagnose and repair the asphalt road hole, no device has been developed for monitoring, scanning and simultaneous repair of the well. Though advanced automotive technology has been invented by the system for scanning the system for coordinating and adapting road suspension systems. But the progress that is being made around the world today is often in the field of preparation, cutting, erosion, leveling, and solidification of asphalt. Of course, the network system has been created to provide intelligent services using electronic transmitters. This design works with the current modified sender, enabling applications to communicate with local transmitters, and exchange information, in the presence of on-line exchanges. To do this research, Caraco has used the structure of the transmitter's signal and its properties in the frequency range (Omid, 2017). If this device can perform all the operations mentioned in a fully automated and intelligent way. Highway gaps are costly and time-consuming. As a result, scientists at the Washington Institute of Technology have developed an automated system that will fill the gap asphalt and increase road life with minimal time and cost. This new approach is a self-priming and automatic filler identification system that connects to an automobile like a wheelchair and uses a stereo camera and light source to detect road gaps. By identifying the gap location, this machine is firmly attached to the site and the vehicle moves at a speed of about $5 \mathrm{~km} / \mathrm{h}$. This system works like a dot matrix printer, which publishes ink points on a moving paper. In the case of the gutter seal system, the road is like a moving sheet of paper, and the nozzles attached to the device throw the material into the slots. Of course, the huge limitation of this device is that it only has the ability to restructure the cracks with the help of the operator. And the results have shown that this system is ultimately capable of detecting only $80 \%$ of road gaps (Echnology, 2007).

Researchers from the Pennsylvania State University have developed a roadmap for road surface repair intelligence, in which the characteristics of gray asphalt surfaces are defined for the safety of road surfaces and drivers' comfort. Currently, road surface and friction measurement is not included in the design of pavement surfaces. To assess road surface features, a locked trailer is used and a laser scale profile is used. A mixed road surface test has also been performed to determine the characteristics of the bulk and mixture properties. The test includes the hardness factor, specific gravity, maximum specific gravity, flame factor, and graded analysis. Mechanical properties of materials have been used to study the effect of dynamic behavior on 


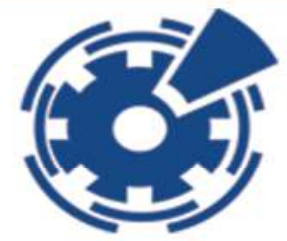

surface properties. Characteristics of road surfaces are measured and calculated using some regression analysis with some common parameters. The properties of pavement coverings have also been analyzed. The analysis variables consisted of 100 tests at $6.4 \mathrm{~km} / \mathrm{h}$ measured using ASTM E501 for smooth surfaces and ASTM E524 for a so-called rubber-like string, with a moderate depth and a gradient of a specific model at Pennsylvania State University (AASHTO, 2007)

There are many research projects in the field of smart asphalt. Smart asphalt has unique advantages. Smart asphalt works in addition to its ability to generate electricity as a link between road and vehicles. So, if smart asphalt is used, the ability to link this invention with the intelligent structure of the road structure will be greater (Robin, 2001).

\section{How to Operate the Machin and Explain?}

To solve the technical problem mentioned above, the best solution is to design a machine that can simultaneously scan and identify the asphalt pavement holes and cavities, and then intelligently cut, clean, and prepare the hole, asphalt, and solid Roll and roll. The purpose of this research is to design the main chassis and platform of a machine that implements all necessary operations for the repair of the pit together with one-time road monitoring Fig. 1 . The device is connected to a deadly car, like a cabin, to provide a driving force needed to operate a diesel fueled car with a capacity of about 200 horsepower. Therefore, all the topics discussed in this study will be presented solely for the cabin trailer.

Figure 1: Chassis and main platform

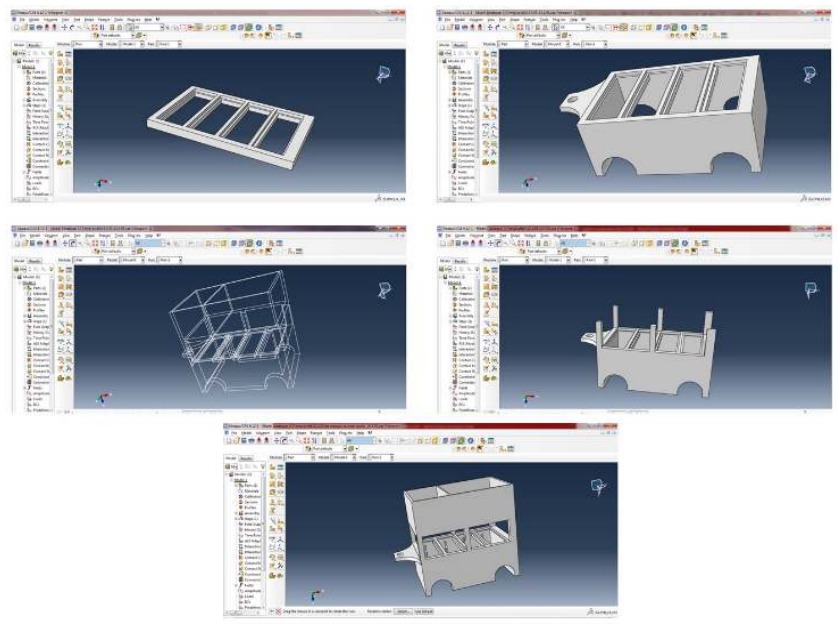

P a g e | 24 


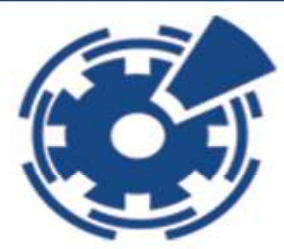

The device consists of three main parts of the sensors, a central processing unit, and executive operators. The sensor set is located at the very first point of the fuselage chassis and scans the whole surface of the road. So that it sends information about the surface, height, and volume of the hole to the central processing unit. Sensors also have the ability to detect road-surface bumps and send out bump information. The central processing unit Fig. 2 , which is a central control unit and processor, receives and processes the information and, according to the pre-algorithm, detects the geometric location of the hole, the surface value, the height, and the volume of the hole. One of the most important pit scan advantages in this way is to register the geometric location of the hole in the electronic systems, which will allow all relevant organizations, if necessary, to have access to this information. For example, insurance companies or car accident experts may decide more closely with this information and declare their opinion on the geometric location of the pits at the event site.

Figure 2: Electronic Control Unit

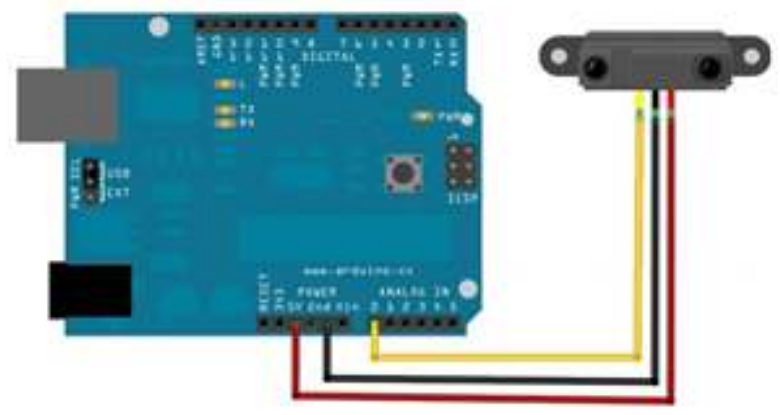

The central processing unit calculates the amount of spatial changes in the operators and the amount of bitumen and asphalt required and the start and end time of each operator's operation. Then, given the results of the processing, it sends commands to each of the operators. The first command is to place the preparation operators in the exact geometric position desired. The solenoid Fig. 3. on the frame of the set of operator actuators guides the frame to the desired geometric location. The analysis of the data sent from the sensors in the central processing unit is carried out with the help of specialized software that has been given to integrated circuit circuits through coding. The geometric location and the exact dimensions of the pit form are converted to a point cloud by grinding and knotting the desired volume by the unit, and then the cloud-dot set will form the total volume of the hole. Therefore, accurate programming and matching of sensor information with orders issued by the processing unit will be very delicate in designing this device. The sensor module discussed in the implementation of this section includes an accelerometer mounted on a front-mounted composite frame, which uses the vibrational properties of a super-specialized Pentium based tablet to enter the general features of the pit. Parameters such as the type of geometric mixture, the connector, the superficial layer, the 


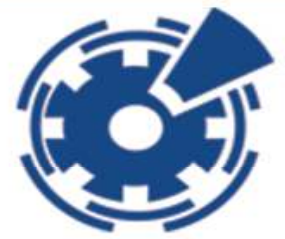

thickness of the layer, the target point density, and the timing system should be sent in real time. The accelerometer uses a three-axis CXL10HF3 accelerometer produced by Crossbow that can measure $10 \mathrm{~g}$ acceleration to $10 \mathrm{kHz}$. The accelerometer also measures roller roll vibrations during condensation. The outer surface temperature is also measured using an infrared temperature sensor mounted on the bottom of the roller bearing frame. Trimble Pro XT GPS Receiver is used to record the momentary position of the roller. This information has been observed in similar investigations reported at the US State Highway Academy (Priyanshu, 2013).

Figure 3: Solenoid Frame Assembly Section Preparation

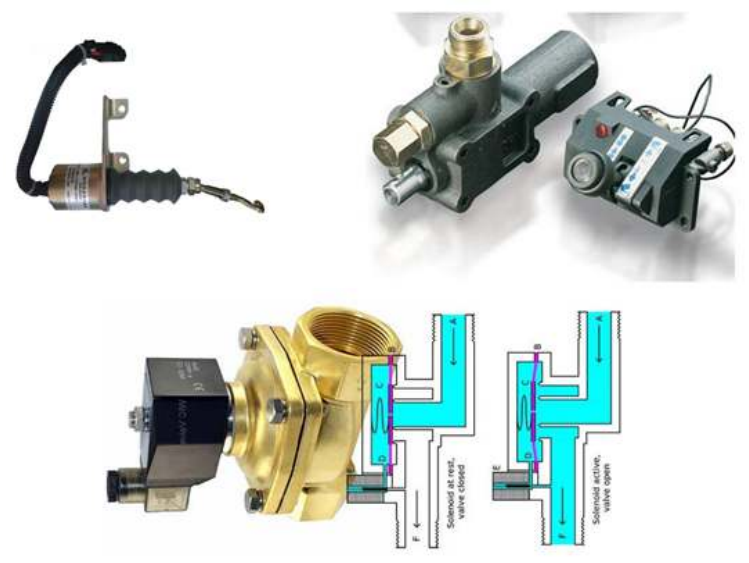

The processing unit issues the command of the start time and end time to the processing unit operators. The most important of these are the asphalt cutter actuator side of the pits, the discharge brush operator, the compressor actuator, and the cleaner of the hole Fig. 4.

Figure: 4 cutting motor operator
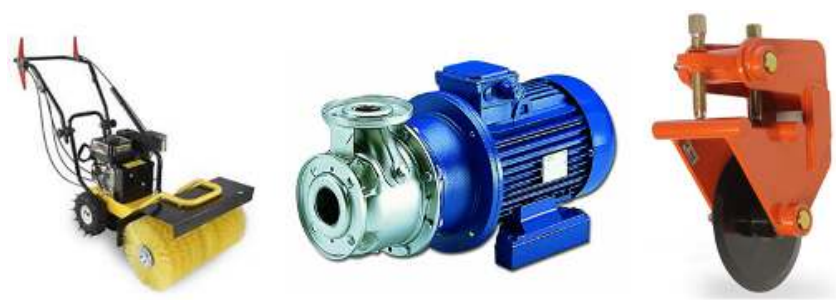

After completion of the operation of the operators of the preparation section, the system of solenoids returns the frame of the set of preparatory operators to the original location. Upon completion of the preparatory phase, the central processing unit sends the transfer order of the frame of nozzle nozzles to the bitumen solenoid. The frame of the nozzle nozzles acts exactly like a rail, which has two degrees of freedom, and can provide bumpers in both the transverse and longitudinal direction. Of course, the design of the pulleys is based on the motor pattern of the stationary motors. In order to precisely spray, the step of the stage motor can be less defined to 


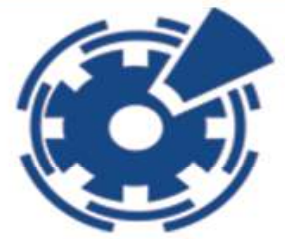

increase spray accuracy. Also, for high-adhesion roadbeds, the step of the stepper motor can be further defined to reduce the amount of spraying. In fact, with the step change of the stage motors, the amount of spray can be controlled and optimized Fig. 5.

Figure 5: Frame assembly section Preparation and nozzle bulking frame
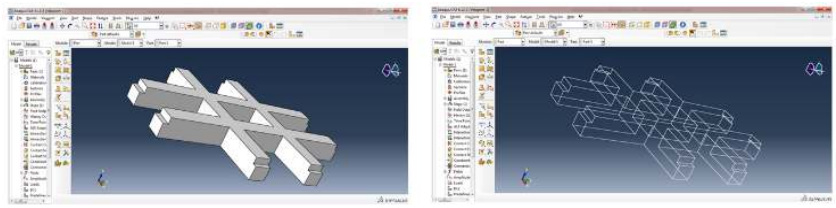

Then the bead frame is placed in a precise geometric location in accordance with the geometric coordinates of the hole, and the nozzles of the powder fill the desired volume of bitumen according to the instructions of the processing unit into the cavity and, after the end of the operation time, the beading frame by the solenoids is directed to its original location It will be. Today, in many countries, new compounds for road pavement are used, which are sometimes in the liquid phase, and asphalt spraying operations are similar to those used for asphalt. These innovative composite materials, which will incorporate bitumen and asphalt compounds in the future, are highly sensitive to changes in environmental conditions, changes in workplace heights, and especially in temperature variations (Technology, 2009). Therefore, the design, construction and production of reservoirs, maintenance equipment, communication tubes and spray nozzles of these materials are also a delicate and highly specialized process. Companies active in the production of equipment and storage tanks are in accordance with the mechanical properties of new materials that can be used to make the invention Fig. 6.

Figure 6: Reservoir and nozzle equipment New Composite Asphalt

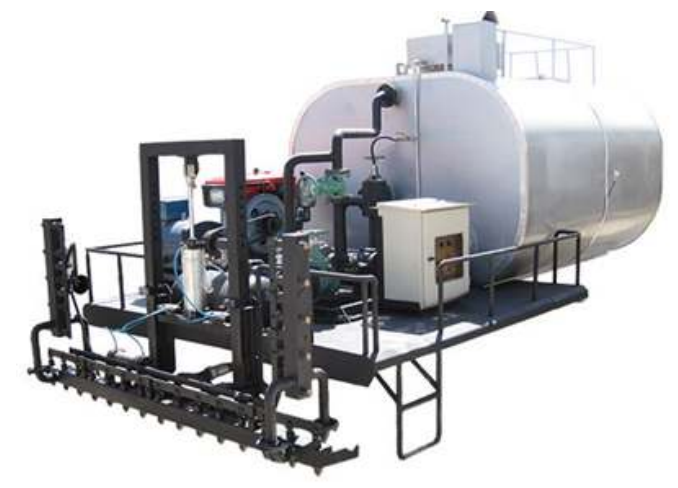

The processing unit will instruct the insertion and drop of the volume of calculated asphalt volume into the frame of the conductor of the asphalt frame and the electric motor valve of the asphalt frame, according to these instructions, will direct the volume of the asphalt from the reservoir to the hole. After the asphalt pouring operation is completed, the asphalt frame will return to its original location. At the final stage of the unit, the processing unit orders the 
placement of the leveling unit, which includes the roller, and the roller after the installation, performs the leveling and reinforcement of the asphalt. Upon completion of the roller time, the order of directing to the original location is issued by the processing unit and the roller will be returned to its original location. The processing unit then sends the message to the driver's monitor to finish repairing the hole, and the driver will find that he will continue to track the next hole to detect. By continuing this cycle, the pits on the asphalt road surface will be fully mechanized. If necessary, urban administrative bodies will be able to control and monitor the operation of the operator and the device by connecting to the central processing unit via the internet.

\section{Conclusion}

Reducing the operation time for road surface diagnosis and repair has a significant impact on the reduction of road traffic in operating areas. The entire restoration process is carried out once on the track of the device on the desired road, so the costs previously spent to track the path to detect, prepare, cut, clean, smear and flatten the asphalt, are spent on The severity decreases. Due to the mechanized and automated operation of the operation, the possibility of error is much less than the traditional implementation. The possibility of electronic recording and archiving of all cavities and road pits for any future research and management is provided. The reduction of risk within the scope of the asphalt restoration workshop is one of the major advantages of the invention compared to the traditional method. Every year, a large number of human resources personnel who are traditionally engaged in the diagnosis and repair of asphalt road pits are at risk of collisions and deaths have occurred. This device is quite intelligent and has only one driver user and the risk is much lower. One of the main advantages of the project is tracking the route, detecting the detection and recording of all paths and pits, integrated path repair and reducing the number and error of manpower. 


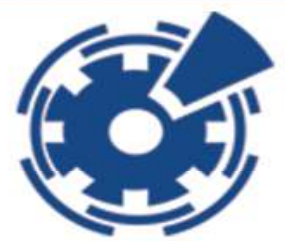

26-28 July, 2019 London, United Kingdom

\section{Acknowledgment}

This invention has been officially registered under the name "Analysis of the smart device invention for the detection and repair of asphalt road surface holes" No. 90629 at the Iranian Invention Office. Hope to find support at this conference

\section{References}

[1] Omid Abari, Deepak Vasisht, Dina Katabi and Anantha Chandrakasan, "Caraoke: An E-Toll Transponder Network for Smart Cities", Massachusetts Institute of Technology Cambridge, MA, USA, Jul 2017.

[2] Echnology Partnerships Program, Federal Highway Administration, Final Report, "Intelligent Asphalt Compaction Analyzer Highways for LIFE", Assistance Agreement DTFH61-08-G-00002, Performance Period October 2007 - December 2009

[3] AASHTO, (2007). AASHTO T-166 Bulk Specific Gravity Of Compacted Bituminous Mixtures Using Saturated Surface-Dry Specimens, Washington 2007.

[4] Robin M. Davis "Comparison of Surface Characteristics of Hot-Mix Asphalt Pavement Surfaces at the Virginia Smart Road" Thesis Submitted to the Faculty of Virginia Polytechnic Institute and State University In partial fulfillment of the requirements for the degree of: Master of Science In Civil and Environmental Engineering, June 22, 2001.

[5] Priyanshu Kumar, "Piezo-Smart Roads", International Journal of Enhanced Research in Science Technology \& Engineering, ISSN: 2319-7463 Vol. 2 Issue 6, June-2013, pp: (65-70), Aligarh Muslim University, Uttar Pradesh, India.

[6] Technology Partnerships Program, Federal Highway Administration, Final Report, "Intelligent Asphalt Compaction Analyzer Highways for LIFE", Assistance Agreement DTFH61-08-G00002, Performance Period October 2007 - December 2009. 